\title{
DYNAMICS WITHIN THE EXCITON FINE STRUCTURE OF COLLOIDAL CDSE QUANTUM DOTS
}

\section{SUPPORTING INFORMATION}

Vanessa M. Huxter, Vitalij Kovalevskij and Gregory D. Scholes

In order to study carrier dynamics, the decay of a spatial population grating was monitored using an ultrafast probe pulse. The full width half maxima (FWHM) and the time bandwidth products (TBWP) of the laser pulses are reported in Table SI. The absorption spectra and the corresponding laser profiles are presented in Figure S1. The transient grating signals discussed in this paper were collected in the phase matched direction corresponding to the wavevector $k_{s}=-k_{1}+k_{2}+k_{3}$ as shown in Figure S2. In addition to the signals previously discussed we also collected time integrated data for the following polarization combinations: VVVV, VVHH, HHVV, VHVH, HVHV, HVVH, VHHV, HHVH, HVVV, VHVV and VVHV in both the $k_{r}$ and the $k_{s}$ signal directions. These polarization combinations comprise the three classes of transient grating signals that are obtained from the various polarization dependencies: normal transient gratings, baselines and the crossed linear polarization signal.

Due to the broad spectral linewidth of the laser pulses as well as the size distribution within the sample, it is possible for different states within the ensemble to be probed by each of the optical interactions. For instance, both pump beams could interact with one particular state while the probe beam interacts with a different state. This situation would be observed as exciton recombination and only contributes to the longtime offset. Alternatively, the pump beams could create a superposition which would provide a contribution that only appears in the coherent spike.

\section{Additional Supporting Measurements}

Additional measurements were performed on a model dye system (Rhodamine 6G in ethanol), CdSe quantum dot samples passivated with TOPO and dispersed in toluene as well as CdSe quantum dot sample passivated with a different ligand (dodecylamine) and suspended in a freestanding PMMA film. The analysis of the dye solution served as a control 


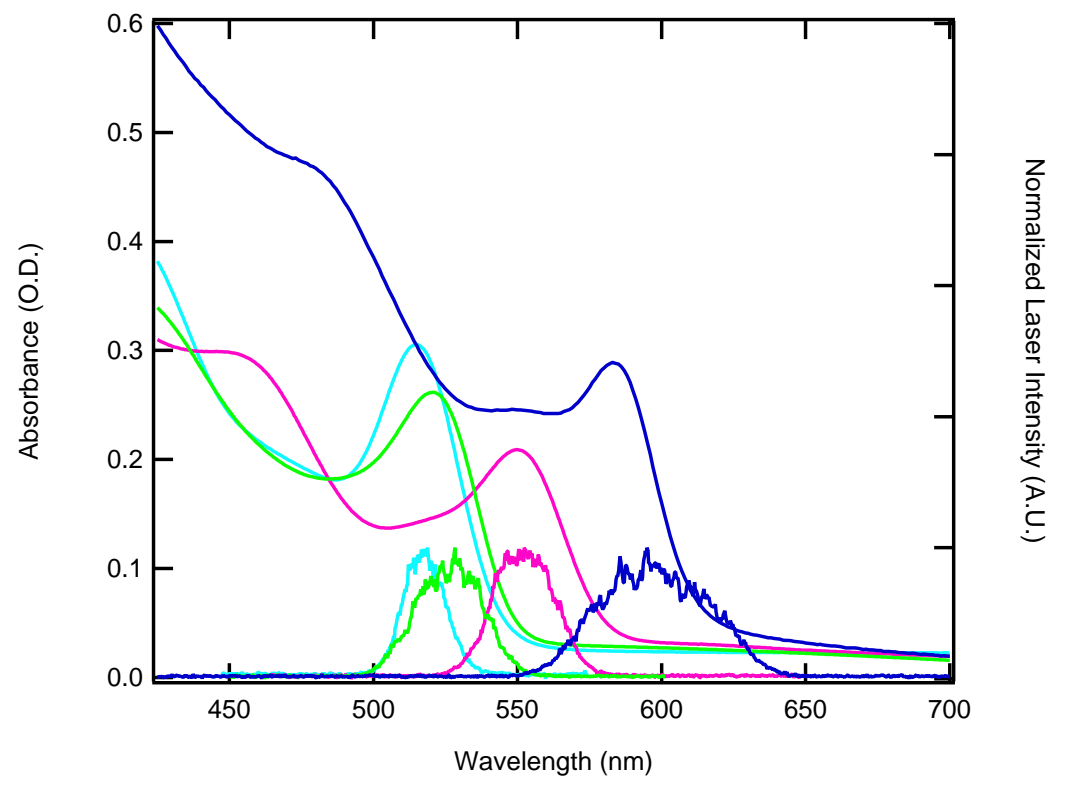

FIG. 1: Absorption and laser spectra for various sizes of CdSe nanocrystal samples passivated with TOPO and embedded in an optically clear polymer film.
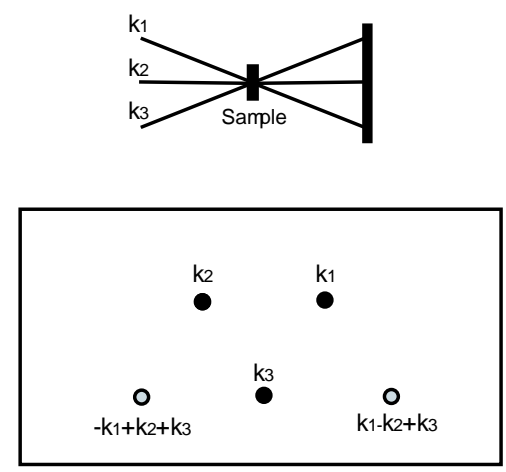

FIG. 2: The beam arrangement for the 3TG experiment is presented schematically. (Top) The incident excitation beams have been split into three components with wavevectors $k_{1}, k_{2}$ and $k_{3}$ generating the transient grating signal which appears at $k_{s}=-k_{1}+k_{2}+k_{3}$ according to phase matching conditions. (Bottom) An in-plane view (perpendicular to the perspective of the top image) of the beams after the sample.

measurement since there should be no difference in the signal observed for the all vertical (VVVV) and the crossed polarization (VHVH) configuration. This was done to ensure that the observed signal was not due to an experimental artifact. The VVVV and VHVH transient gratings in the $k_{s}=-k 1+k 2+k 3$ signal direction for the dye are presented in Figure S3. 
TABLE I: VHVH Polarized Transient Grating Experimental Parameters for CdSe Passivated with TOPO in a Freestanding Polymer Film

\begin{tabular}{llll}
\hline \hline Nanocrystal Radius(nm) & Center Wavelength(nm) & FWHM(fs) & TBWP $^{a}$ \\
\hline 1.55 & 515 & 36 & 0.61 \\
1.70 & 530 & 25 & 0.56 \\
1.95 & 550 & 24 & 0.57 \\
2.50 & 590 & 20 & 0.62 \\
\hline \hline
\end{tabular}

${ }^{a}$ Assuming Gaussian pulse envelopes

The CdSe solution sample was analyzed to verify that there is no significant contribution attributable to the polymer matrix. Similarly, a CdSe quantum dot sample passivated with a different ligand (dodecylamine) and suspended in a PMMA film was analyzed to study the possible surface state contributions to the observed response. The VVVV transient gratings for a CdSe sample capped with TOPO in a polymer film, a CdSe sample capped with TOPO dispersed in a toluene solution and a CdSe sample capped with dodecylamine in a polymer film are presented in Figure S4(a). The comparison is complicated a little by the fact that the CdSe nanocrystal cores in each of the three samples presented were not exactly the same size. The absorption maxima for the amine capped sample corresponded to 523nm, the absorption maxima for the sample in toluene solution corresponded to $525 \mathrm{~nm}$ and the absorption maxima for the sample in a polymer film was 530nm. The VHVH transient gratings for the three CdSe samples are presented in Figure S4(b). The difference in surface state contributions between the CdSe samples passivated with TOPO and the one passivated with dodecylamine are clearly visible in the VVVV trace, however, the time scales are short enough in the VHVH trace that the surface states do not significantly contribute. The samples (CdSe capped with TOPO) analyzed in solution versus the samples analyzed in polymer film do not show any significant differences. 


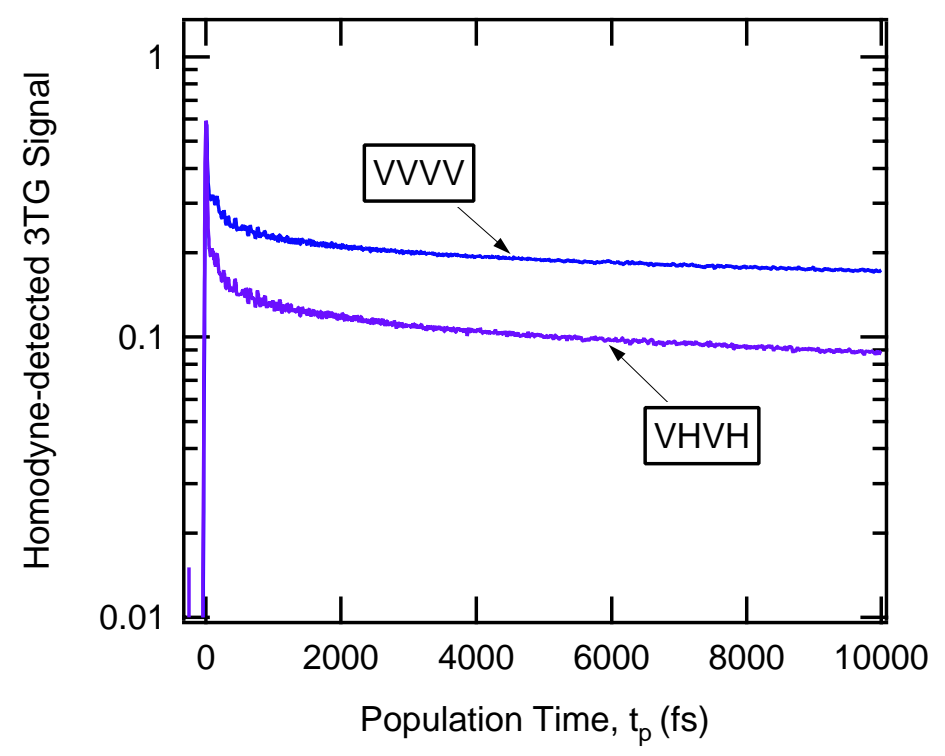

FIG. 3: Transient grating traces measured in the $k_{s}=-k_{1}+k_{2}+k_{3}$ of a Rhodamine-6G dye solution in ethanol plotted on a semi-log scale. The top trace corresponds to the VVVV polarizer configuration while the bottom trace corresponds to VHVH.
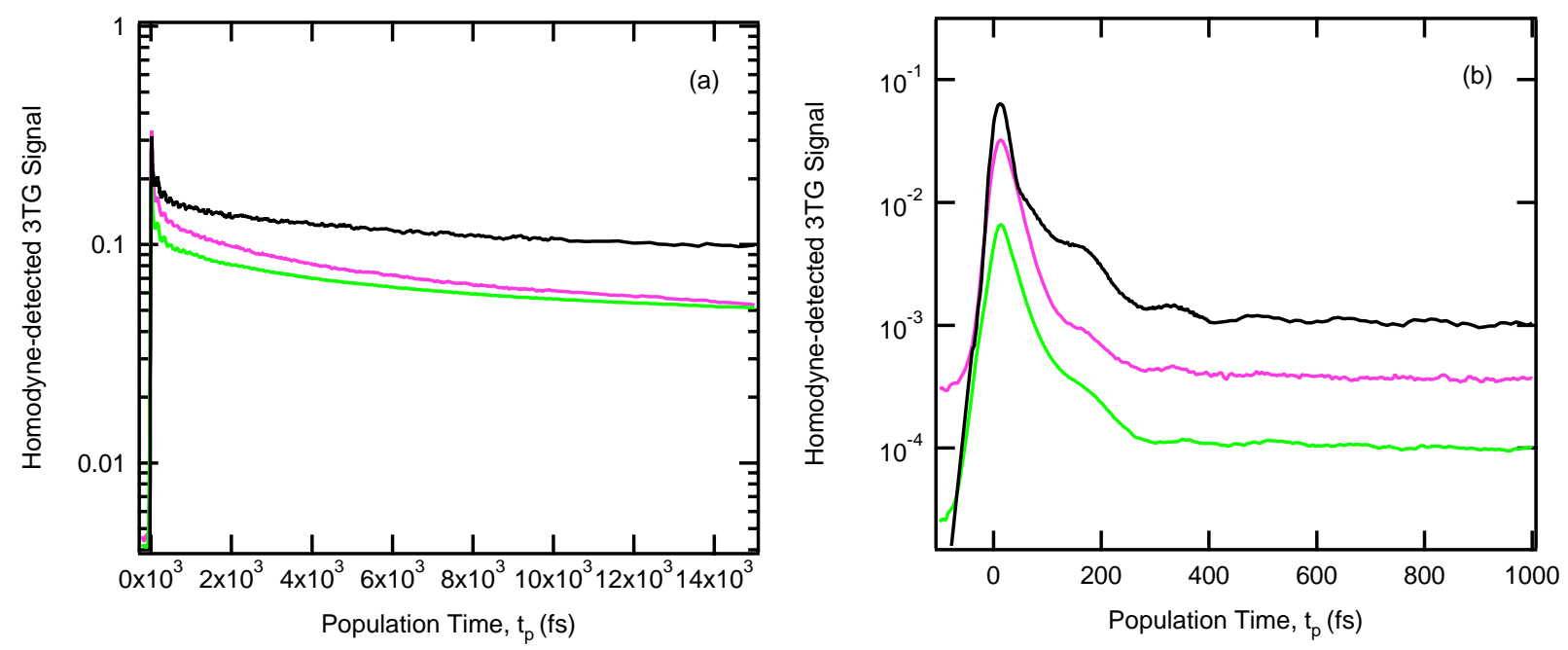

FIG. 4: Transient grating traces plotted on a semi-log scale obtained in $k_{s}=-k_{1}+k_{2}+k_{3}$ direction for the (a) VVVV polarization combination and the (b) VHVH polarization combination. In both (a) and (b) the top trace (black) corresponds to the CdSe capped with TOPO in a polymer film (absorption peak centered at 530nm), the middle trace (pink) corresponds to the dodecylamine capped CdSe (absorption peak at 523nm) and the bottom trace (green) corresponds to the CdSe capped with TOPO in a toluene solution (absorption peak at 525nm). The traces have been offset for clarity. 